\begin{tabular}{|c|c|c|}
\hline Beitr. Ent. & Keltern & ISSN 0005-805X \\
\hline $\mathbf{5 5}(2005) 2$ & S. $485-498$ & 27.12 .2005 \\
\hline
\end{tabular}

\title{
Myrmecology in the internet: Possibilities of information gathering
}

\section{(Hymenoptera, Formicidae)}

with 12 tables

Christiana Klingenberg and Manfred Verhaagh

\begin{abstract}
Summary
A well advanced information system about ants exists on the internet. Many myrmecologists all over the world offer useful information as text files, images or databases. A good part of the information focuses on regional faunas and biogeographic regions, with updated species checklists and geographic distribution maps. Many internet sites deal with specific ant groups or single genera and provide dichotomous and sometimes interactive identification keys, extensive information about the biology and/or geographic distribution of the species. Text information is often illustrated with images of living or dry mounted ants. Additionally, detailed pages about anatomy, mounting of ants, colony husbandry or ant conservation (red lists) can be found. In discussion forums it is possible to exchange facts and thoughts about all myrmecological facets with other interested people. A special offer for taxonomists is the increasing number of databases about museum collections and their type catalogues.
\end{abstract}

\section{Zusammenfassung}

Für Ameisen gibt es mittlerweile ein sehr gut entwickeltes Informationssystem im Internet. Weltweit bieten zahlreiche Myrmekologen brauchbare Informationen in Form von Textbeiträgen, Bildern und Datenbanken an. Ein guter Teil der Information beschäftigt sich mit regionalen Faunen bzw. biogeographischen Regionen, z.B. in Form von Artenlisten und Verbreitungskarten. Viele Internetseiten handeln auch spezielle Ameisentaxa ab, stellen dichotome oder gar interaktive Bestimmungsschlüssel vor und offerieren ausführliche Informationen zur Biologie und Verbreitung der Arten. Die Texte werden häufig durch Bilder präparierter oder lebender Ameisen ergänzt. Es lassen sich auch detaillierte Seiten über Anatomie, Präparation, Haltung und Schutz finden. Diskussionsforen bieten die Möglichkeit, sich über alle Aspekte der Myrmekologie mit Gleichgesinnten auszutauschen. Ein wichtiges Angebot speziell für taxonomisch Interessierte ist die zunehmende Zahl von Sammlungs- und Typenlisten.

\section{Keywords}

ants, Formicidae, internet based information, database, type images 


\section{Introduction}

The World Wide Web is the most dynamic information source about any given topic. Performing internet searches about "ants" in different languages, the popular search engine "Google" presents close to 3.5 million records. But not all of the recorded internet sites are dealing in a reliable or useful manner with ants (BARONI URBANi \& ANDRADE 2003). The aim of this publication is to give a list of major and helpful internet sites about ants within a broad spectrum of themes that, however, will be far from exhaustive.

\section{Results}

We distinguish different categories of sites, the limits, however, are often far from being sharply drawn. There are some sites that serve as kind of internet portals about ants (table 1). Beside own information, these sites offer links to other pages with many different topics. The main site in this category is "Antbase", initiated and maintained by Donat Agosti and Norman F. Johnson. It is the pilot site of the Social Insects World Wide Web (SIWeb) and aims to give all available information about ant names (via "Hymenoptera Name Server") and primary taxonomic literature (citations and full text copies); there are links to important other databases, a glossary about morphological terms and the directory of world's ants taxonomists.

The main topic of another category of highly useful internet pages is taxonomy and systematics (table 2). These pages are partly integrated into the ant portals. "Antweb" initiated by Brian Fisher of the California Academy of Sciences provides superb high resolution Automontage ${ }^{\odot}$ images of nearly all ant genera of the world. The "Hymenoptera Name Server" offers the actual taxonomic status of any given species name with its synonymies and original descriptions, often available for download in PDF file format. In this category also fits the webpage of the "Tree of Live"-project. This site deals with phylogenetic (cladistic) relationships among all taxa on earth and also gives a short description of the natural history of the requested organism, with useful links to sites with more specific information and images.

Sites about the content of collections and type catalogues are available in growing number. Some examples are given in table 3. The homepage of the Museum of Comparative Zoology at Harvard University is a very good example because the authors provide high resolution images of deposited ant types. The German project "Digital information on ant collections in Germany and their types (FoCol)" within the GBIF-D program has the same focus (Verhaagh \& Kuingenberg 2004). High resolution images of the ant types in German collections and any other available information such as taxonomic status and original literature will be available on the international GBIF homepage from 2006 on.

Very important and helpful are websites with ant literature (table 4). The database "Formis" has a key function in this respect; one can search for ant literature published since 1650. Weblogs have a message board character containing also links to new publications. "Notes from Underground" is an informal ant newsletter established since three years in the internet, formerly published in paper prints. It also offers a message board. 
There are many websites related to regional ant faunas (table 5). A majority of pages is dealing with the North American ant fauna. The Japanese Image Database gives detailed information about the ant fauna of each Japanese island. Similar pages are available about the ant fauna of other countries and continents, but in very different completeness. Most of these pages are providing checklists, identification keys, distribution maps, images often in excellent quality, literature, glossaries, hints for specimen preparation and link lists. In a few cases information about natural history is given, too. Outstanding in this category are the web pages of John Longino about Costa Rican ants and of Steve Shattuck about Australian ants.

For several ecologically important ant groups such as leaf cutting ants or fire ants web sites with detailed information on their natural history are available (table 6). They often include images of living ants and links to related sites.

A category of ant related web sites of growing importance deals with invasive ants (table 7). In most of the presented sites those ants are not only treated as pests in the context of human activities, but also as ants which are dangerous for the native ant fauna. Identification guides are given, often illustrated with images. Geographic distribution maps on regional level are also available in some cases. Useful information on the biology of invasive species may also be found on some sites about controlling pest ants. But we made no attempt to check the very high number of websites that deal with pest ant control.

There are a few web sites about endangered ants (table 8). The IUCN red list is also linked to antbase.org. The German "Ameisenschutzwarte" deals mainly with Formica spp. and provides information about activities in red wood ant conservation and literature, and maintains a discussion forum.

Glossaries are helpful especially for beginners in myrmecology (table 9). At some of the web sites related to regional ant fauna and internet portals (e.g. "Antbase") glossaries are given. Two other sites listed here not only give a list of explained terms, but the main structures are illustrated and detailed descriptions of each structure with links to the main morphological complex are given.

URLs to images of mounted and living ants are given in table 10 . To our opinion the web page of Alex Wild "myrmecos.net" shows the best images of living ants we have seen in the web. The superb pictures of mounted specimens of all ant genera at "Antweb" have already been mentioned above under the taxonomic category. The Australian Ant Image Database is actually a subsite of the Japanese Ant Image Database. Both sites provide very good images of mounted and living ants. Many other pages, especially on sites of regional ant fauna (see above) also provide images of ants in high resolution quality.

For people who like to maintain living colonies at home or under laboratory conditions useful hints are given on the sites listed in table 11 . Discussion forums about ant husbandry are available, too. The ecological risks for the environment and the native fauna in the case that exotic ant species escape from captivity should be kept in mind (Buschinger 2004). Serious precautions against escapement should accompany any rearing of exotic ant species that nevertheless should be restricted to responsible scientific investigations.

Discussion forums in different languages are the last category of our ant related web sites (table 12). There is more than one discussion forum in German. Links to them can be found on other sites, such as "Ameisenschutzwarte" or "Ameisenhaltung". 


\section{Conclusion}

The links presented here are only a small subset of all available web sites which deal with ants. We have chosen these sites, because they seem to be maintained regularly and all URLs have been verified by us. Many of these sites hold links to other sites and bear more information than we have commented.

It became evident that most web sites are maintained by US-American colleagues, or at least with support of them. European or German web sites about ants with comparative quality are still quite rare.

A difference to printed information is that web sites are more or less always under construction and sometimes not available (there are also many "dead" links). The contributions are not peer-reviewed - except for information copied from peer-reviewed texts. Thus, one should be ready to read really silly ideas like "Preventive measures [against ants]: Destroy all Hymenoptera's nests around your home" (from the site "Poisonous animals and plants - Ants"; http://library.thinkquest.org/C007974/2 4ant.htm) Internet sites offer today only a small part of all the information available on ants. They cannot replace traditional information sources as already stated by BARONI URBANI \& ANDrAde (2003) but can complement them to growing extent.

\section{Acknowledgement}

We thank Jochen Bihn for critical reading of the manuscript and the hint to the link "Poisonous animals and plants".

\section{Literature}

Baroni Urbani, C. \& Andrade, M. L. de 2003: The ant genus Proceratium in the extant and fossil record. - Museo Regionale di Scienze Naturali, Torino, Monografie XXXVI, 496 pp.

Buschinger, A. 2004: Risiken und Gefahren zunehmenden internationalen Handels mit Ameisen zu PrivatHaltungszwecken (Hymenoptera, Formicidae). - Myrmecologische Nachrichten 6: 79-82.

VerhaAgh, M. \& Klingenderg, C. 2004: Digital information on ant collections in Germany and their types (FoCol). - In: Berendsohn, W. G. \& Oehlschlaeger, S. (Hrsg.): GBIF-D: German participation in the Global Biodiversity Information Facility - Status Report 2004. Bonn: PT-DLR Environmental Research and Technology \& Berlin: Botanic Garden and Botanical Museum Berlin-Dahlem, 84-85.

After submission of our paper the following publication appeared which offers few additional web resources about ants: NASH, D. R. 2005: Web resources for myrmecologists. - Myrmecologische Nachrichten 7: 95-102.

\section{Author's addresses:}

Dipl. Biol. Christiana Kuingenberg \& Dr. Manfred Verhaagh

Staatliches Museum für Naturkunde Karlsruhe

Erbprinzenstr. 13

D-76133 Karlsruhe

e-mail: klingenberg@smnk.de; manfred.verhaagh@smnk.de; http://www.smnk.de/ 


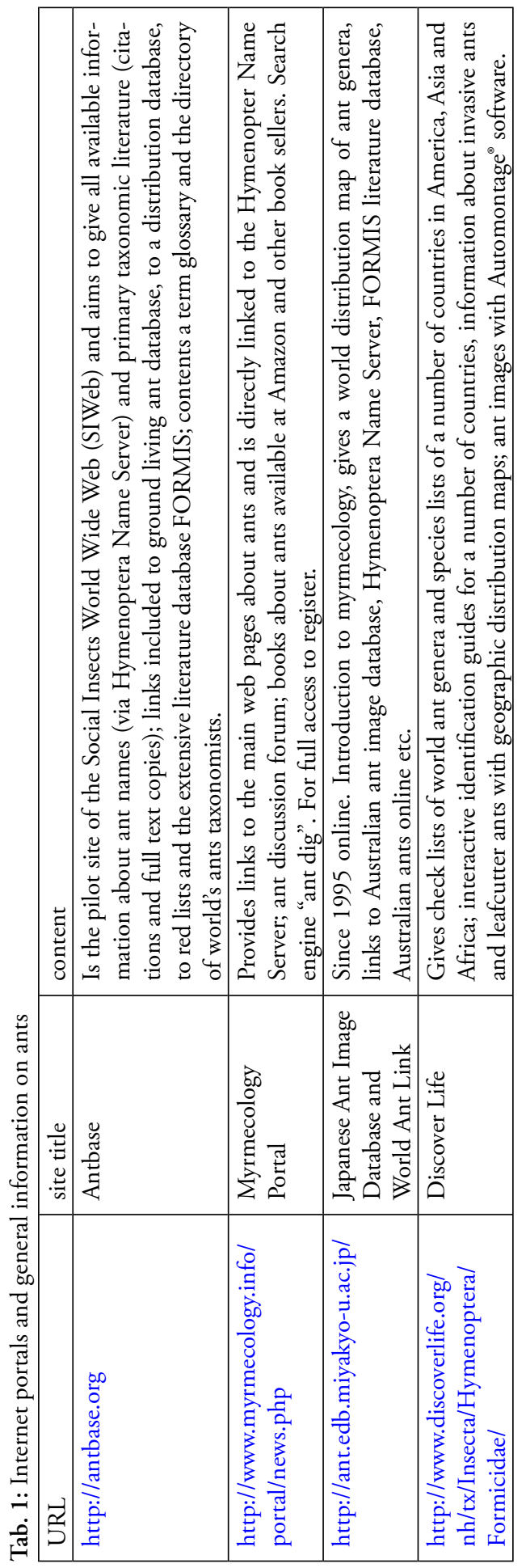

\begin{tabular}{|c|c|c|c|c|c|}
\hline 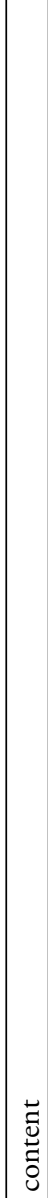 & 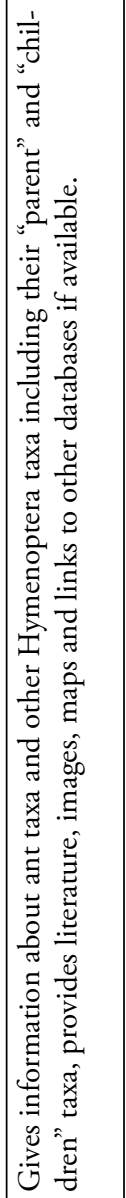 & 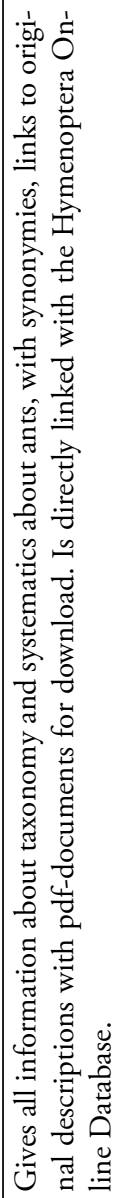 & 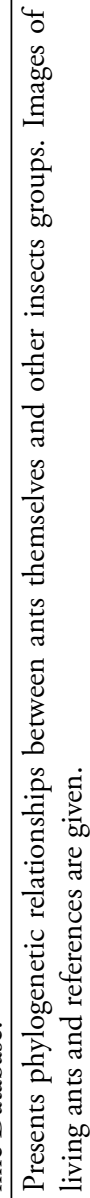 & 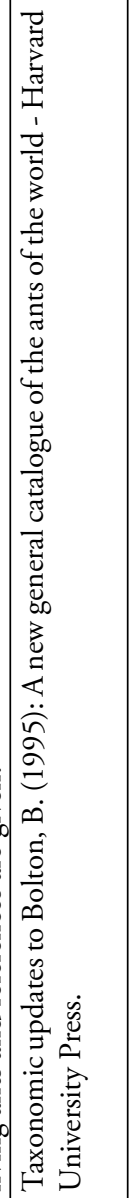 & 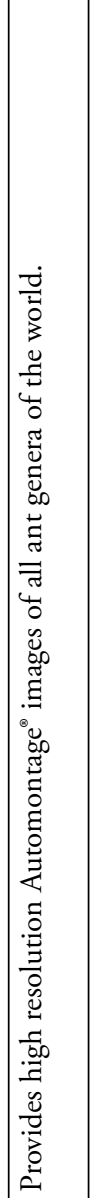 \\
\hline 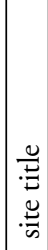 & 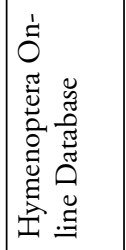 & 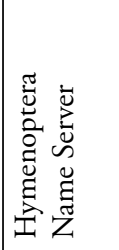 & 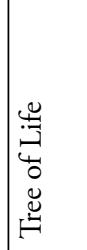 & 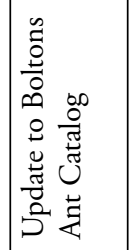 & 离 \\
\hline 孚 & 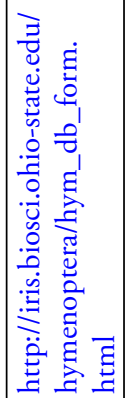 & 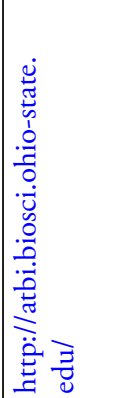 & 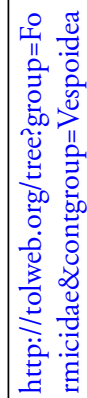 & 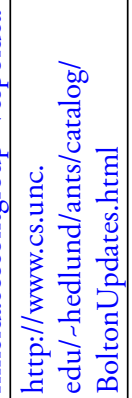 & 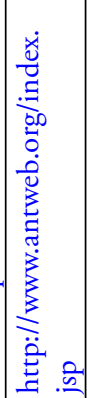 \\
\hline
\end{tabular}




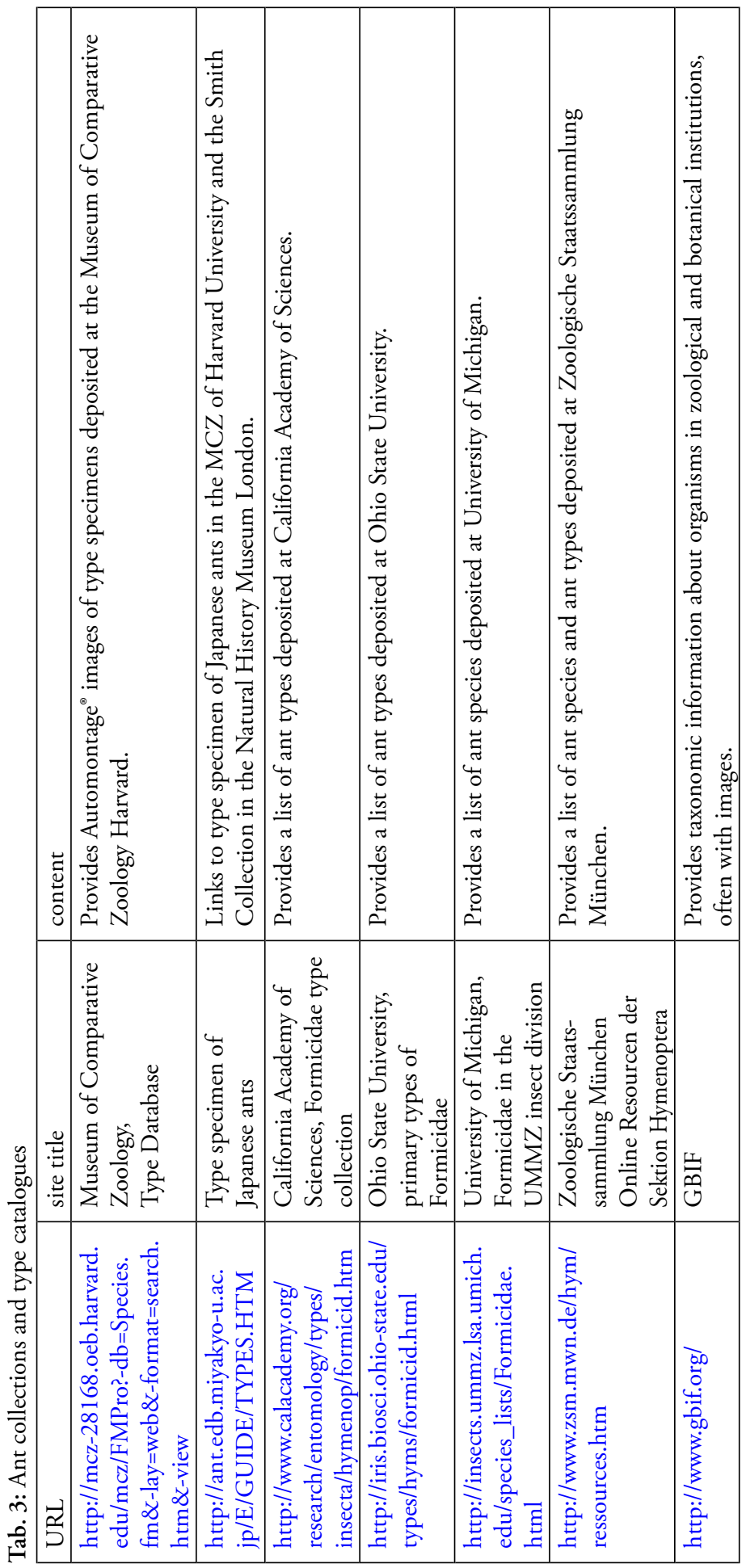




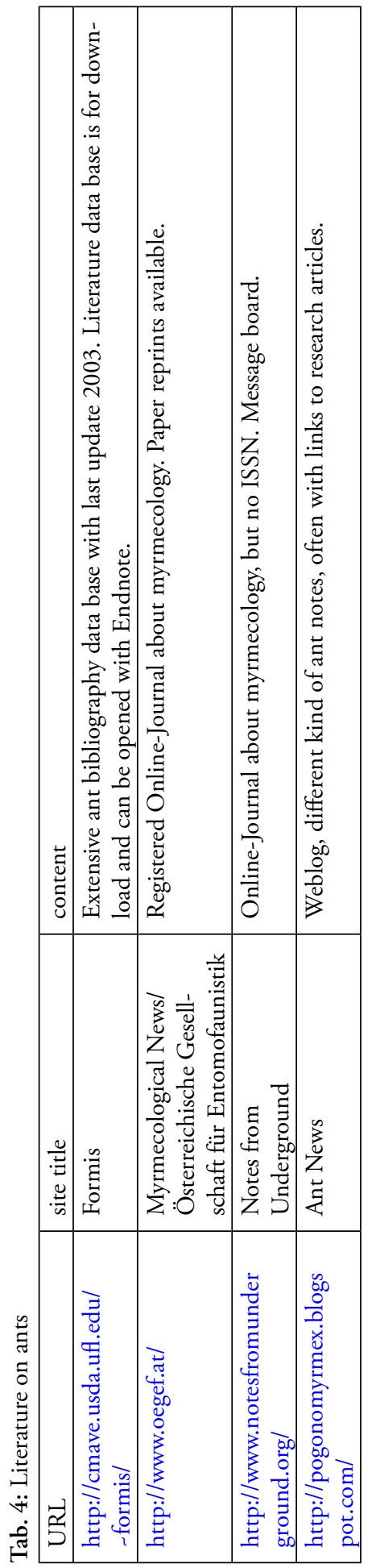

\begin{tabular}{|c|c|c|c|c|c|}
\hline 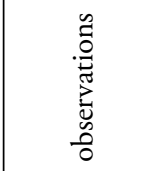 & 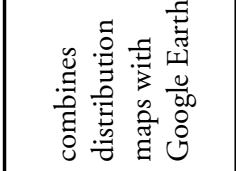 & & & 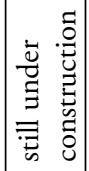 & \\
\hline$\stackrel{y}{\Xi}$ & & + & & & + \\
\hline 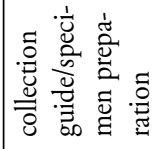 & & & & & \\
\hline 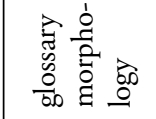 & & & & + & \\
\hline $\begin{array}{l}\mathscr{8} \\
\tilde{\Xi} \\
\Xi\end{array}$ & 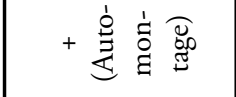 & & + & 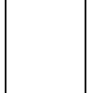 & + \\
\hline 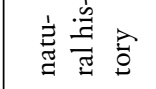 & & & + & + & + \\
\hline 离 气 & & & + & & \\
\hline 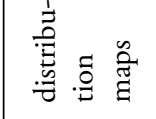 & + & & + & & \\
\hline 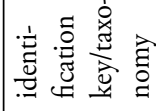 & & & & + & \\
\hline 离 & + & + & + & & + \\
\hline . & 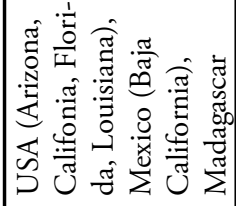 & 岕 & 芯 & 峁 & 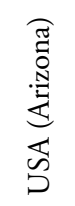 \\
\hline 冬 & 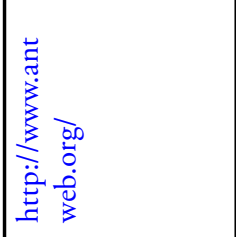 & 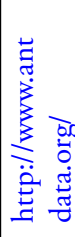 & 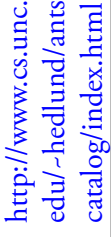 & 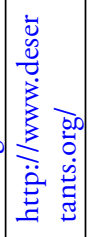 & 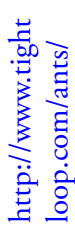 \\
\hline
\end{tabular}




\begin{tabular}{|c|c|c|c|c|c|c|c|}
\hline 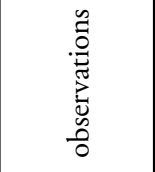 & 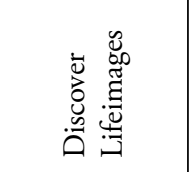 & & & & & 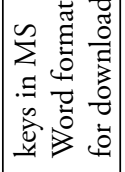 & \\
\hline$\stackrel{\tilde{g}}{\Xi}$ & + & + & & & & + & + \\
\hline 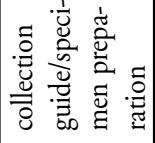 & & + & & & & & \\
\hline 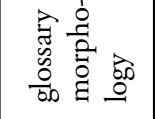 & + & & & & & & \\
\hline 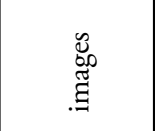 & + & + & & 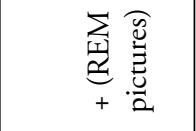 & & 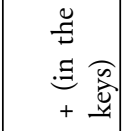 & + \\
\hline 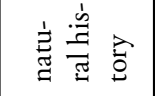 & & & & + & + & & \\
\hline 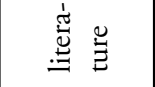 & & + & & + & + & + & \\
\hline 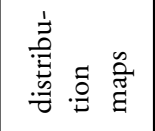 & & + & & & & 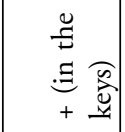 & \\
\hline 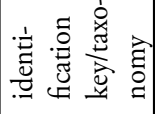 & + & + & & & + & + & \\
\hline 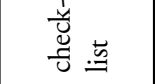 & + & + & + & + & + & & + \\
\hline . & 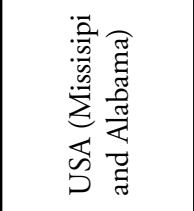 & 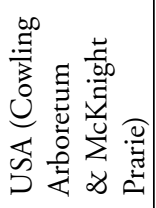 & 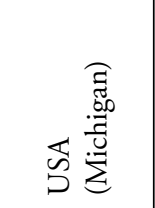 & 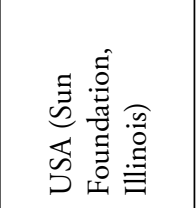 & 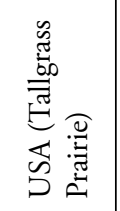 & 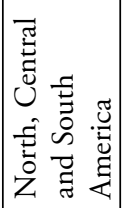 & 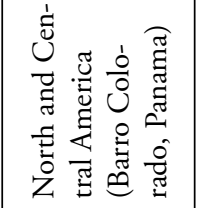 \\
\hline 몸 & 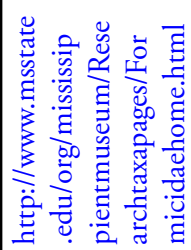 & 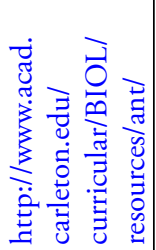 & 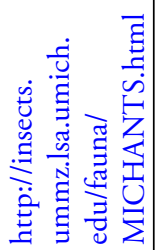 & 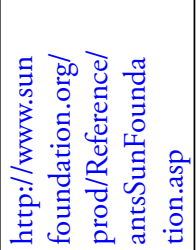 & 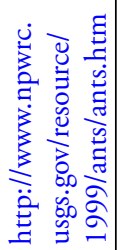 & 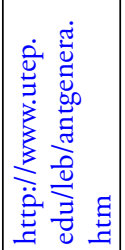 & 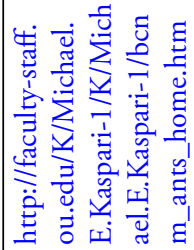 \\
\hline
\end{tabular}


Beitr. Ent. 55 (2005) 2

\begin{tabular}{|c|c|c|c|c|c|c|c|c|c|}
\hline 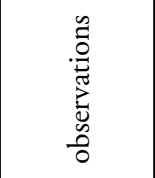 & 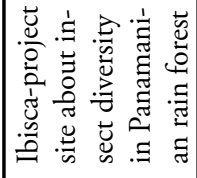 & & 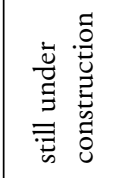 & & & & & & \\
\hline$\stackrel{\ddot{\Xi}}{\Xi}$ & & + & + & & & & & + & + \\
\hline 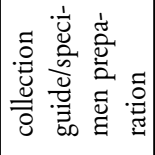 & & + & + & & & + & & & \\
\hline 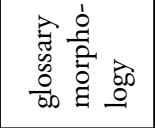 & & & & & & + & & + & + \\
\hline 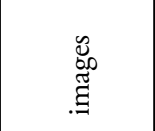 & $\begin{array}{l}+\frac{1}{2} \widehat{g} \\
+\frac{\tilde{g}}{0}\end{array}$ & + & & + & & 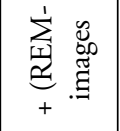 & + & + & \\
\hline 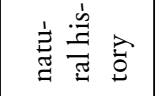 & + & + & & & & + & & + & + \\
\hline 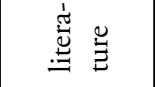 & + & + & + & + & & + & + & + & + \\
\hline 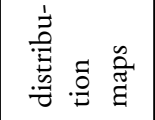 & & & & + & & + & & + & \\
\hline 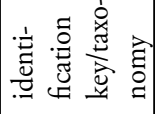 & & + & & + & + & + & & + & + \\
\hline 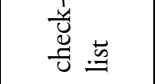 & & + & & + & & + & + & + & + \\
\hline$\cdot \frac{5}{0}$ & 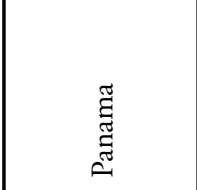 & 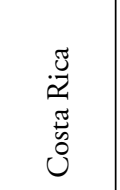 & $\begin{array}{l}\overline{\widetilde{N}} \\
\text { ص్ }\end{array}$ & 递 & 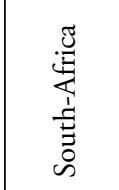 & 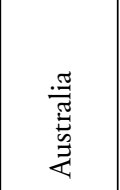 & 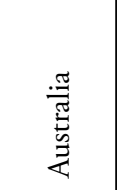 & 㽕 & $\begin{array}{l}\text { :ี } \\
\text { के }\end{array}$ \\
\hline 㞘 & 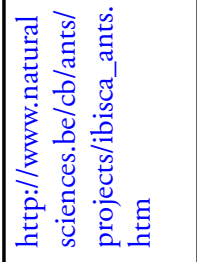 & 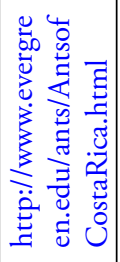 & 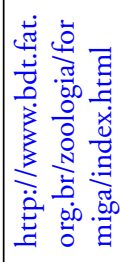 & 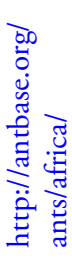 & 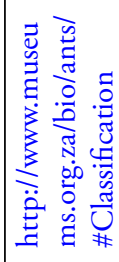 & 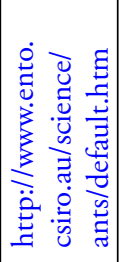 & 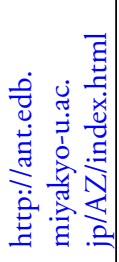 & 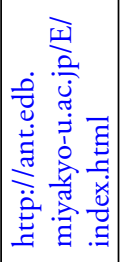 & 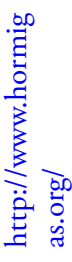 \\
\hline
\end{tabular}




\begin{tabular}{|c|c|c|c|c|c|}
\hline 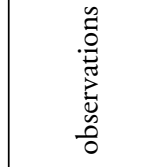 & & 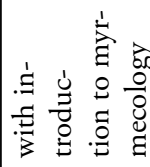 & 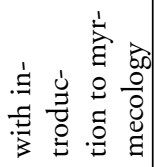 & & \\
\hline$\stackrel{\ddot{n}}{\Xi}$ & & + & + & & \\
\hline 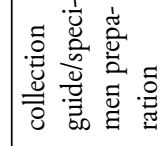 & & & & & \\
\hline 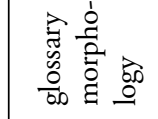 & & + & & & \\
\hline 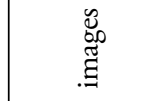 & & + & + & & + \\
\hline 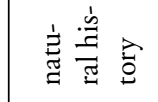 & & + & + & + & \\
\hline 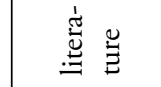 & + & & & + & + \\
\hline 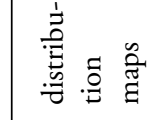 & & & & + & \\
\hline 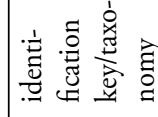 & & + & & & \\
\hline $\begin{array}{l}\frac{\dot{y}}{\mathscr{U}} \\
\frac{\tilde{U}}{U}\end{array}$ & 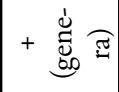 & + & & + & + \\
\hline. & 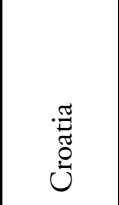 & 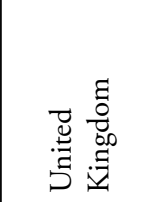 & 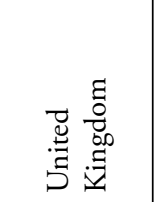 & 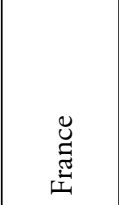 & 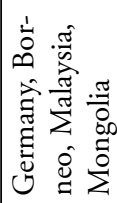 \\
\hline 로 & 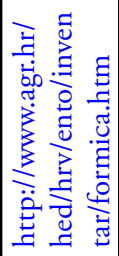 & 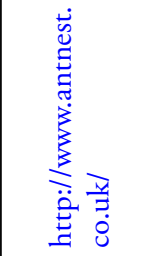 & 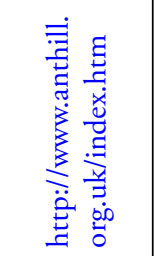 & 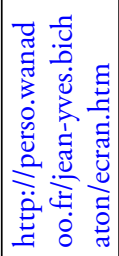 & 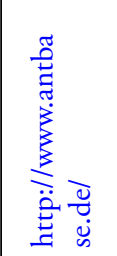 \\
\hline
\end{tabular}

\begin{tabular}{|c|c|c|c|c|}
\hline & 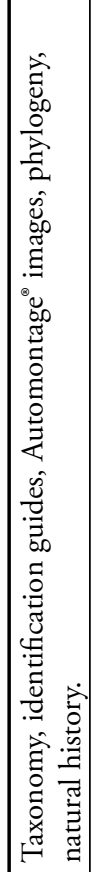 & 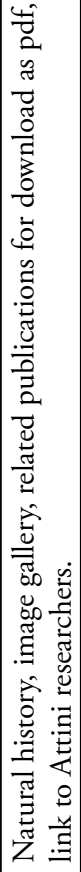 & 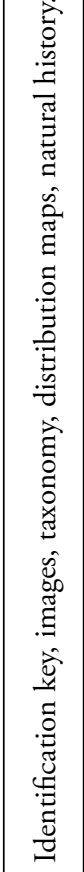 & 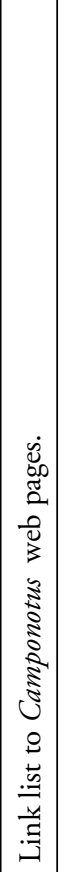 \\
\hline 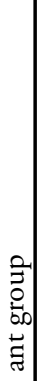 & 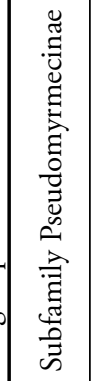 & 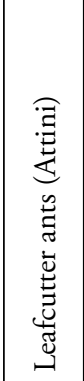 & 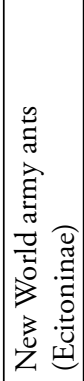 & 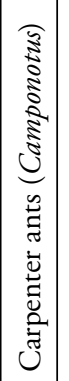 \\
\hline 占 & 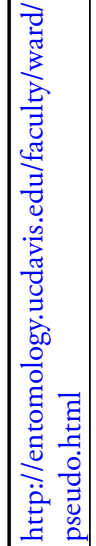 & 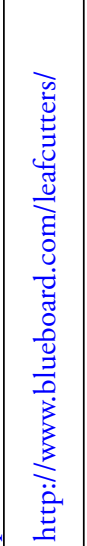 & 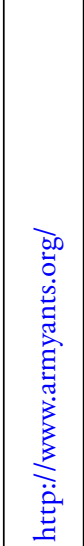 & 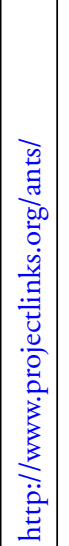 \\
\hline
\end{tabular}



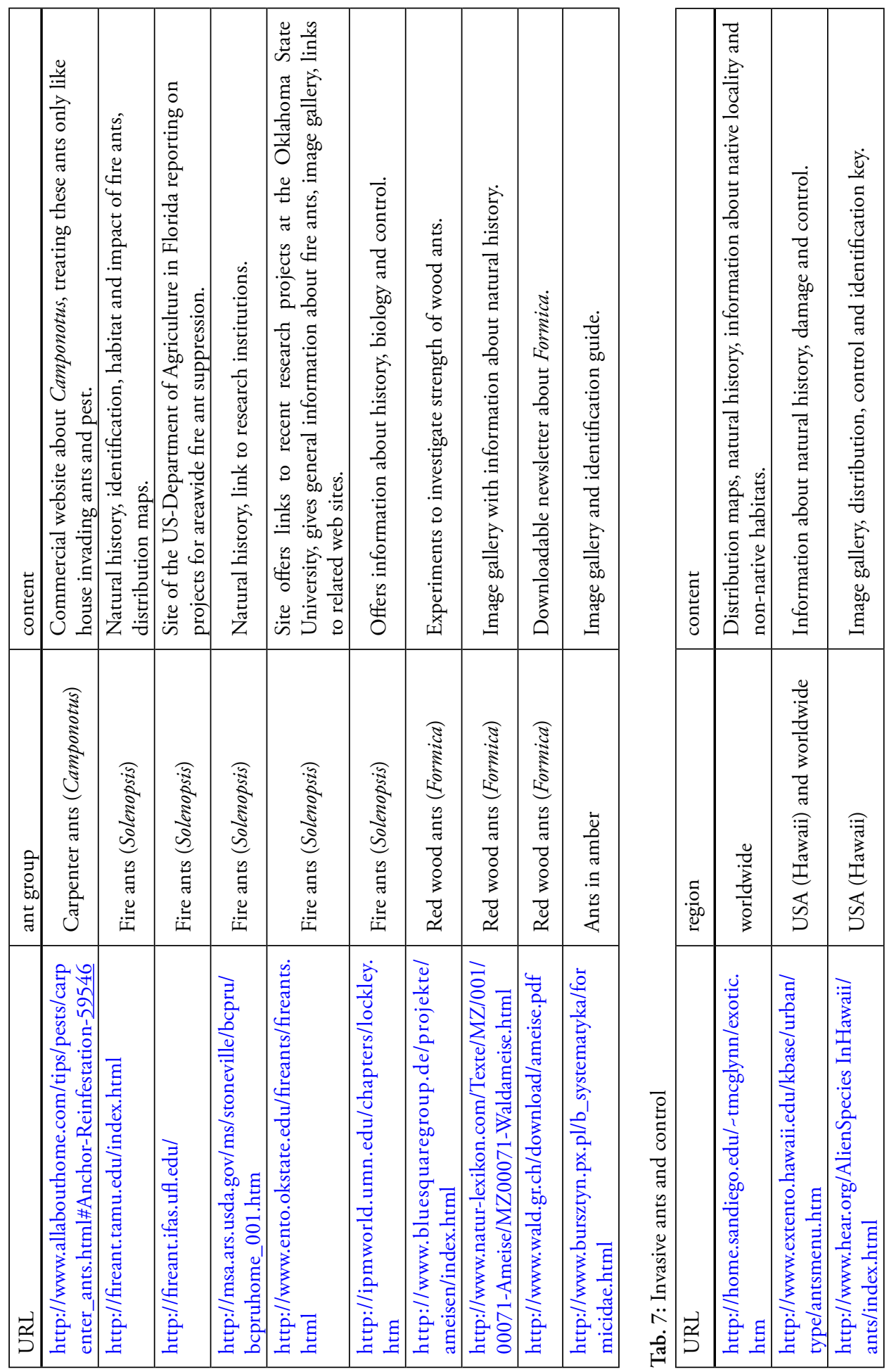

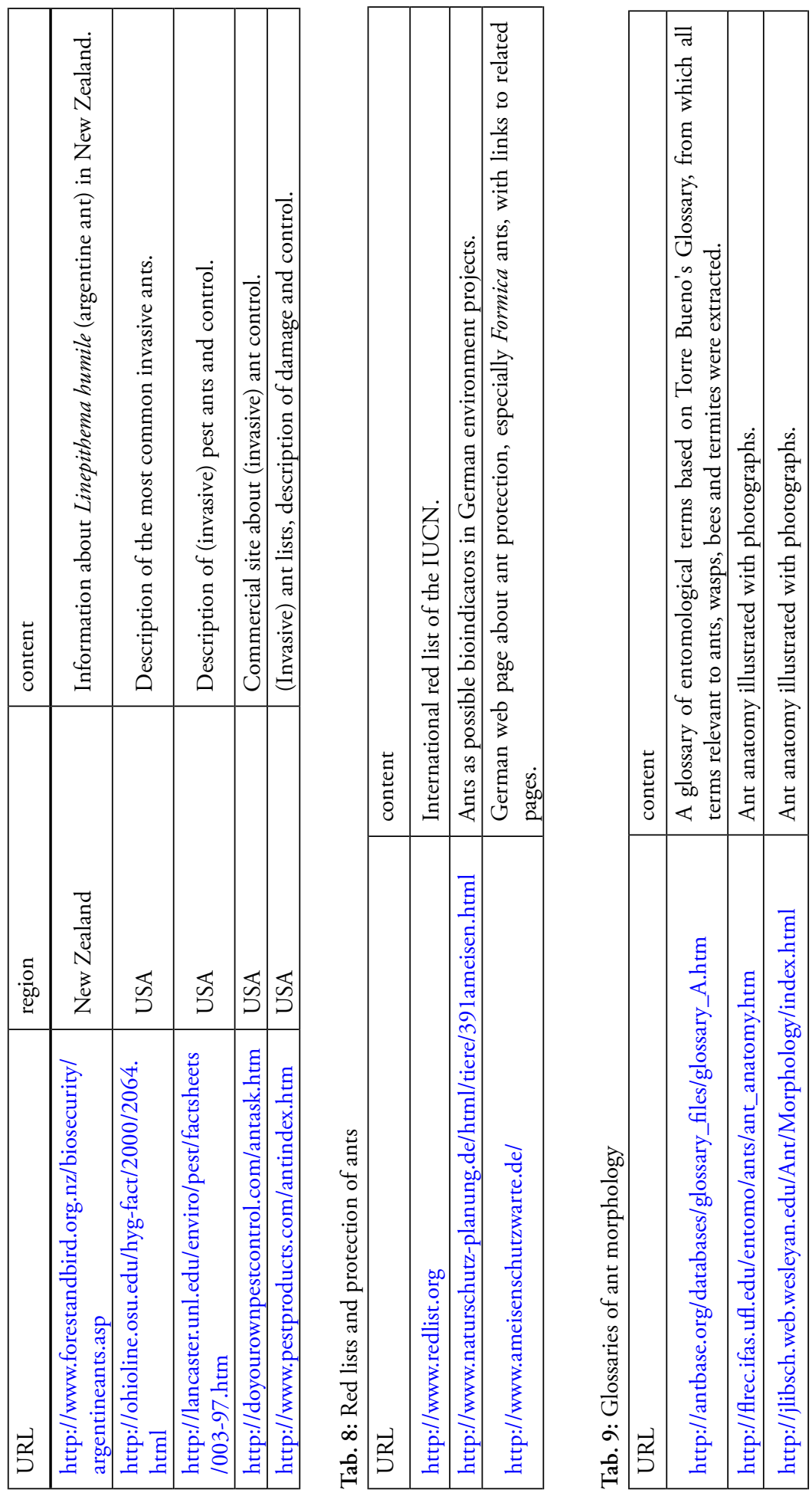

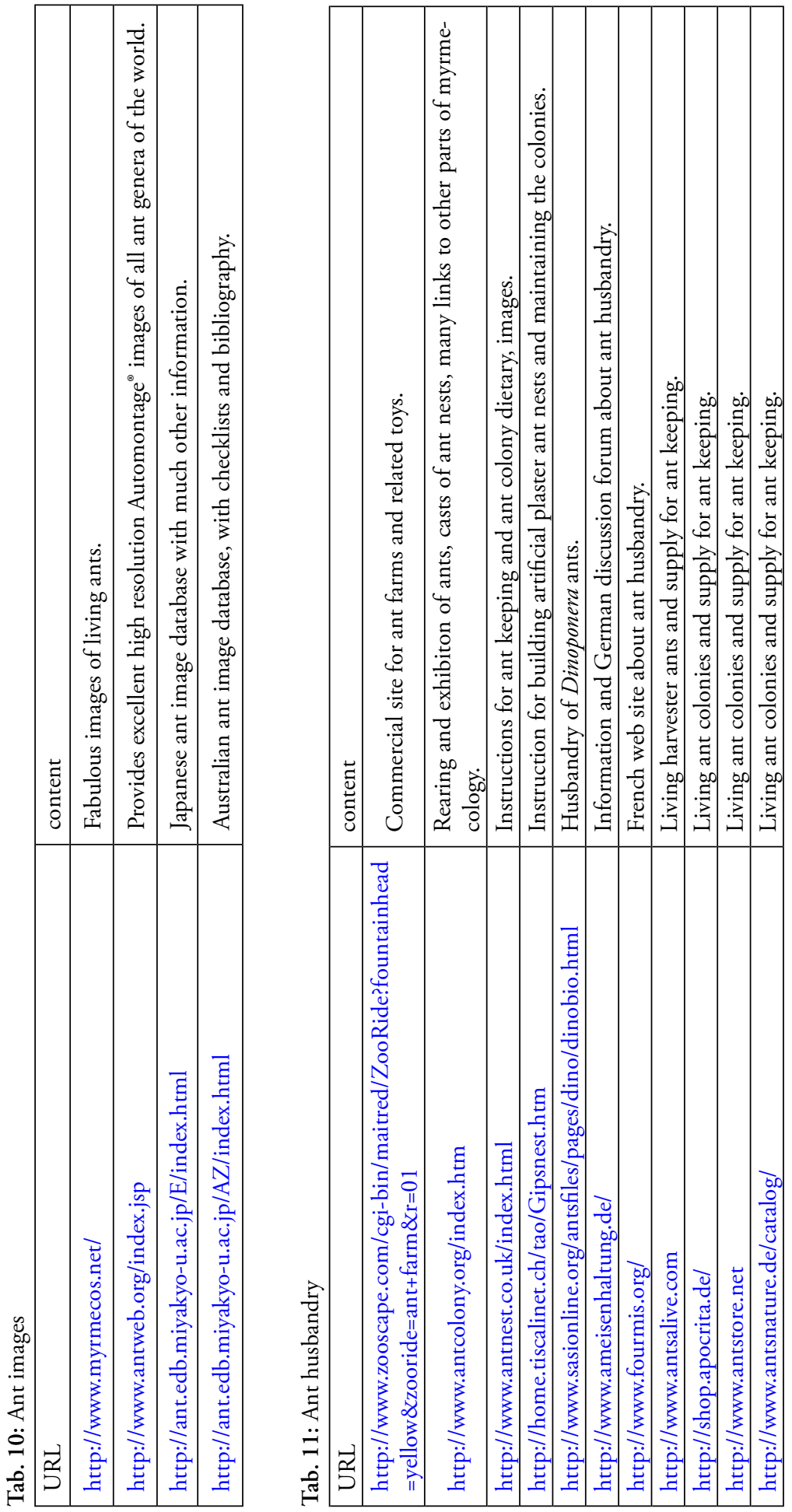
Tab. 12: Ant forums

\begin{tabular}{|l|l|}
\hline URL & language \\
\hline http://www.lamarabunta.org/ & Spanish \\
\hline http://www.ameisenforum.de & German \\
\hline http://www.ameisenschutzwarte.de/forum/index.php & German \\
\hline http://www.ameisenhaltung.de/forum & German \\
\hline http://groups.yahoo.com/group/antcolonies/messages & English \\
\hline http://rufaman.proboards15.com/index.cgi & English \\
\hline http://p211.ezboard.com/bantfarm & English \\
\hline
\end{tabular}

